III.

\title{
A Fibroid Polypus weighing about one pound and a half, growing from the left lip of the Cervix Uteri.
}

\author{
By James Oliver, M.D., F.R.S. (Edin.), \\ Physician to the Hospital for Women, Soho.
}

N.H., æt. 33, a virgin, was sent to the Hospital for Women by Dr. Edward Morris, of East Croydon, on July 15th, and Dr. Morris was present at the operation when I removed the tumour by morcellattion. The history given of the ease was that until Christmas, 1907, menstruation had recurred regularly and the discharge was ordinary in amount. Since Christmas menstruation had recurred at intervals of six or seven weeks and the discharge was accompanied by clots. About the end of January, or five and a half months before seeking advice, the patient remarked that she was unable to retain her urine, and this condition of affairs persisted until July 13th when, on account of inability to pass urine, she consulted Dr. Morris. The bladder was then found to be enormously distended and sixty ounces of urine were drawn off by means of the catheter. After July 13 th there was again incontinence. On account of the bulk of the neoplasm it was impossible to reach the cervix and the relationship to the cervix was only determined after the greater portion of the growth had been removed by morcellation. The tumour sprang from the left lip of the cervix and did not encroach upon the os. I so cut the base of the tumour that I obtained two flaps and these, when stitched together, left the os uteri still undisturbed. The tumour was composed of very coarse, pearly-looking strands of fibrous tissue, and the capsule was so incorporated with these strands that morcellation of the capsule had to be effected with the underlying structure. During the operation the patient lost a very large quantity of blood and became collapsed. The pieces of tumour, numbering about twenty, weighed ono pound and a quarter, and as these were ex-sanguine, the tumour in its original state must have weighed one pound and a half or possibly one pound and three-quarters.

Diagram 1, depicts the tumour in the vagina. Diagram 2, depicts the stumps after the flaps had been stitched together with cat-gut. 


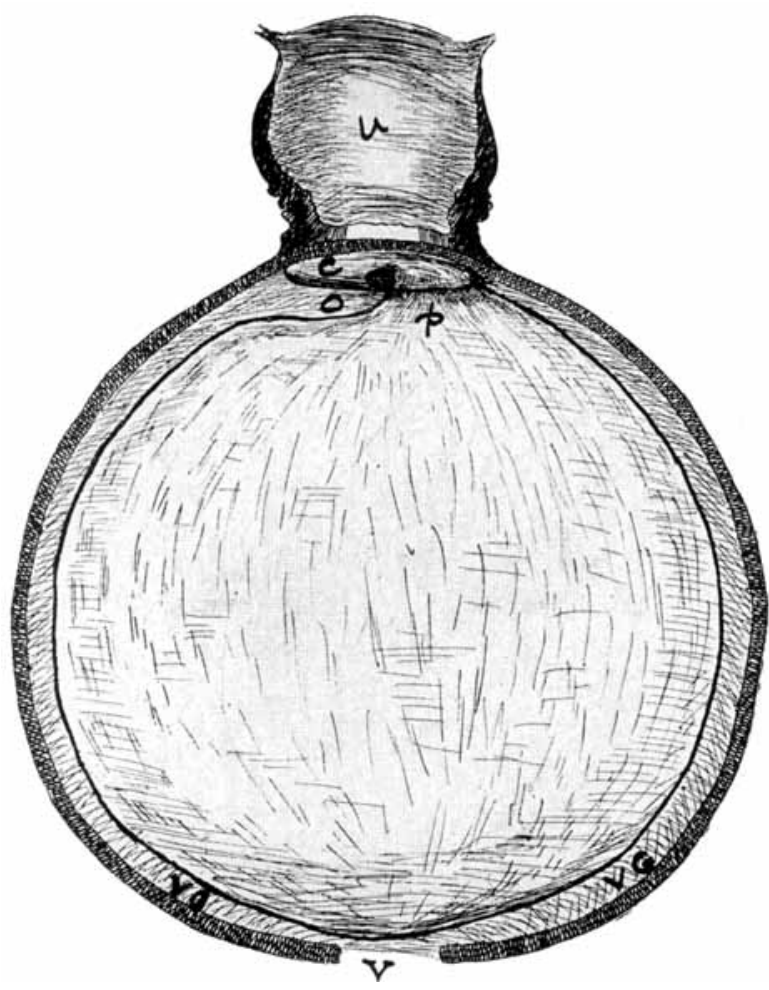

Fig. I.

U. Uterus.

C. Cervix.

o. Close to os uteri.

$P$. On very short pedicle.

V.G. Vaginal canal.

V. Vaginal orifice.

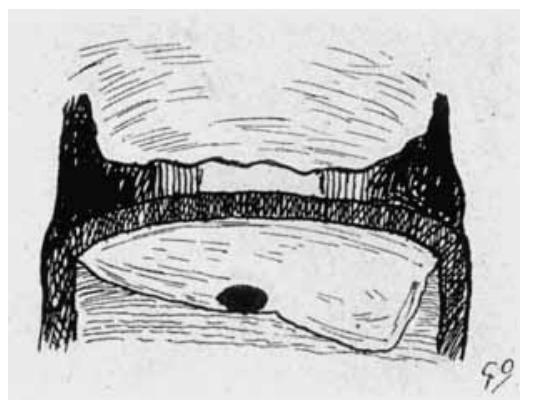

FIG. II.

Showing stump of left lip of cervix after operation. 